\title{
Terapias de infusión en la enfermedad de Parkinson avanzada
}

\author{
Cassandra Navarro-Roa ${ }^{1}$, Mayela Rodríguez-Violante ${ }^{1}$ y Amin Cervantes-Arriaga ${ }^{1,2}$ \\ ${ }^{1}$ Clínica de Trastornos del Movimiento; ${ }^{2}$ Laboratorio Clínico de Enfermedades Neurodegenerativas. Instituto Nacional de Neurología y Neurocirugía, \\ Ciudad de México, México
}

\begin{abstract}
Resumen
La enfermedad de Parkinson (EP) es un trastorno neurodegenerativo cuyo tratamiento es sintomático, habitualmente iniciado como monoterapia, pero con la progresión de la sintomatología y aparición de complicaciones motores es frecuente el uso de politerapia. El paciente con enfermedad avanzada con respuesta inadecuada al tratamiento con fármacos orales o transdérmicos puede ser candidato a terapias asistidas por dispositivo como la estimulación cerebral profunda, la apomorfina en infusión subcutánea continua (AISC) o la levodopa/carbidopa intestinal en gel (LCIG). Estas dos últimas también reciben la denominación de terapias de infusión. La presente revisión tiene el objetivo de presentar los antecedentes históricos, características farmacológicas, indicaciones, contraindicaciones, inicio y dosificación, efectividad sobre síntomas motores y no motores, perfil de seguridad y selección del candidato ideal de las terapias con AISC y con LCIG para el paciente con EP avanzada.
\end{abstract}

Palabras clave: Enfermedad de Parkinson avanzada. Terapia asistida por dispositivo. Terapia de infusión. Apomorfina en infusión subcutánea continua. Levodopa/carbidopa intestinal en gel.

\section{Título en inglés: Infusion therapies in advanced Parkinson's disease}

\section{Abstract}

Parkinson's disease (PD) is a neurodegenerative disorder whose treatment is symptomatic, usually initiated as monotherapy, but with the progression of symptoms and the appearance of motor complications the use of polytherapy is frequent. The patient with advanced disease with an inadequate response to treatment with oral or transdermal drugs may be a candidate for device-assisted therapies such as deep brain stimulation, continuous subcutaneous apomorphine infusion (CSAI) and levodopa/carbidopa intestinal gel (LCIG). The last two treatments also receive the denomination of infusion therapies. The present review has the objective of presenting the historical background, pharmacological characteristics, indications, contraindications, initiation and dosage, effectivity on motor and non-motor symptoms, safety profile and selection of the ideal candidate for CSAI and LCIG therapies for patients with advanced PD.

Key words: Advanced Parkinson's disease. Device-assisted therapy. Infusion therapy. Continuous subcutaneous apomorphine infusion. Levodopa/carbidopa intestinal gel.

\section{Correspondencia:}

Mayela Rodríguez-Violante

Clínica de Trastornos del Movimiento

Instituto Nacional de Neurología y Neurocirugía

Insurgentes Sur, 3877

Col. La Fama

Fecha de recepción: 24-01-2018

Fecha de aceptación: 17-11-2018

C.P. 4269, Ciudad de México, México

E-mail: mrodriguez@innn.edu.mx

DOI: 10.24875/RMN.M19000018
Disponible en internet: 20-03-2019 Rev Mex Neuroci. 2019;20(1):3-13 www.revmexneurociencia.com 1665-5044/@ 2019. Academia Mexicana de Neurología A.C. Publicado por Permanyer México. Este es un artículo Open Access bajo la licencia CC BY-NC-ND (http://creativecommons.org/licenses/by-nc-nd/4.0/). 


\section{Introducción}

La enfermedad de Parkinson (EP) es la segunda enfermedad neurodegenerativa más común. La incidencia de la EP aumenta con la edad y se estima que la prevalencia oscila entre 217.22 y 308 por cada 100,000 personas $^{1,2}$. En México no existen datos sobre prevalencia, pero de acuerdo con la Dirección General de Epidemiología la incidencia en 2017 fue de 10.26 por 100,000 personas.

La EP se caracteriza clínicamente por temblor, rigidez, bradicinesia e inestabilidad postural; además de estos hallazgos motores existen también manifestaciones no motoras de la enfermedad, tales como alteraciones neuropsiquiátricas, disautonomías, alteraciones del sueño, gastrointestinales y sensitivas. Las complicaciones no motoras pueden, incluso, discapacitar más al paciente de lo que lo hace la afectación motora.

El tratamiento más efectivo para los síntomas motores de la EP es la levodopa; la cual se administra en conjunto con inhibidores periféricos de la dopa-descarboxilasa (carbidopa o benserazida). Otros tratamientos disponibles, tales como inhibidores de la monoaminooxidasa $B$ (selegilina o rasagilina), inhibidores de la catecol-O-metiltransferasa (entacapona) y agonistas dopaminérgicos no ergóticos (pramipexol o rotigotina), tienen la finalidad de aumentar la disponibilidad de dopamina; y a pesar de tener resultados más sutiles en el tratamiento de los síntomas motores pueden coadyuvar al tratamiento de síntomas no motores ${ }^{3}$.

Las etapas avanzadas de la EP se caracterizan por la aparición de fluctuaciones tanto motoras como no motoras. Así mismo, estas etapas se caracterizan por presencias de ON (presencia de efecto sintomático del tratamiento) con discinesias importantes y periodos de OFF (ausencia de efecto sintomático del tratamiento) cada vez más severos. Lo anterior deteriora la calidad de vida de los pacientes, en los cuales los periodos de ON son cada vez más cortos ${ }^{4}$.

La fisiopatología de estas fluctuaciones motoras no se ha dilucidado completamente, pero involucra la pérdida cada vez mayor de dopamina y la modificación de sus receptores. Por otra parte, también se presenta sobreactividad del sistema glutamatérgico, mayor cantidad de ácido gamma-aminobutírico (GABA) en el globo pálido interno y de receptores serotoninérgicos, de cannabinoides y adrenérgicos. Por otra parte, la fisiopatología de las discinesias incluyen fluctuaciones de las concentraciones de dopamina, hipersensibilidad de las sinapsis corticoestriatales y exceso de liberación de GABA $^{5}$.
Una vez que las estrategias farmacológicas convencionales con antiparkinsonianos orales 0 transdérmicos son insuficientes para la gestión de las complicaciones asociadas al tratamiento, el manejo de pacientes con EP avanzada implica el empleo de terapias avanzadas, o más recientemente denominadas terapias asistidas por dispositivo. Estas terapias asistidas por dispositivo incluyen la estimulación cerebral profunda (ECP), así como dos terapias de infusión continua. El presente artículo tiene la finalidad de describir las características e indicaciones de la apomorfina en infusión subcutánea continua (AISC) y la levodopa/carbidopa intestinal en gel (LCIG) ${ }^{6}$.

\section{Apomorfina}

La apomorfina es una molécula que se ha utilizado desde el 2000 a.C. En la civilización Maya se utilizaba un extracto de la raíz de Nymphaea caerulea, la cual contiene apomorfina y se usaba como parte de rituales religiosos ${ }^{7}$.

La apomorfina es un derivado no narcótico de la morfina descubierto en 1869 por Matthiessen y Wright, quienes separaron dos átomos de hidrógeno y un átomo de oxígeno de la molécula de morfina, para así producir un nuevo alcaloide, la apomorfina, la cual en un inicio fue utilizada como emético. En 1884, Wiel sugirió por primera vez el uso de apomorfina en el tratamiento de la EP, puesto que para esas fechas el medicamento se había probado ya en pacientes con epilepsia y corea. En 1951, Schwab describió el efecto benéfico de la apomorfina en pacientes con EP, a los cuales notó la mejoría de la rigidez y del temblor tras la administración subcutánea de 0.6-0.9 mg del medicamento. En la década de los setenta Cotzias trató a pacientes que previamente habían respondido a la terapia dopaminérgica con EP con apomorfina y mostró que la apomorfina tenía una potencia similar a la levodopa en la respuesta motora, sin embargo su administración oral se acompañaba de efectos adversos serios como la uremia. En 1988, Stibe describió la infusión continua subcutánea de apomorfina para reducir los periodos de OFF en los pacientes con EP8.

\section{Farmacología de la apomorfina}

La apomorfina estimula los receptores D1, D2, D3, D4 y se considera un agonista dopaminérgico potente, con un efecto comparable al de la levodopa. Tiene una estructura de amina policíclica terciaria, altamente lipofílica, lo cual le confiere propiedades similares a las de la dopamina y le permite el paso a través de la 
barrera hematoencefálica. La biodisponibilidad oral del medicamento es muy baja debido al metabolismo de primer paso, además de poseer un pobre perfil de tolerabilidad por esta vía. De forma subcutánea, la apomorfina se absorbe rápidamente después de la inyección, en 15-20 minutos, con una biodisponibilidad del $100 \%$. Su vida media es de 30 a 60 minutos $^{9}$.

\section{Vía de administración}

A la fecha, la apomorfina solo está disponible para su administración subcutánea mediante un dispositivo de pluma para rescate ${ }^{10}$ y una bomba de infusión subcutánea. En este artículo se abordará solamente el uso de terapia en bomba de infusión continua.

\section{Indicaciones y selección del paciente}

Como en cualquier terapia, la efectividad radica en la selección adecuada del paciente. Se consideran candidatos para recibir AISC aquellos que tras ser evaluados por un neurólogo o especialista en trastornos del movimiento cumplan con al menos una de las siguientes indicaciones terapéuticas ${ }^{11,12}$ :

- Pacientes con EP avanzada con fluctuaciones motoras ON-OFF incapacitantes y que no se logran controlar adecuadamente con la optimización del tratamiento oral.

- Pacientes con EP que respondan a la apomorfina administrada con pluma, pero que requieran más de cuatro a seis rescates al día.

- Pacientes con EP que permanecen con discapacidad a pesar de ECP.

- Paciente con EP que presenten dificultades para la deglución que interfiere con la adecuada toma de medicamentos.

- Pacientes con EP y problemas gastrointestinales serios que retrasen o limiten la absorción de medicamentos orales.

Adicionalmente, es imprescindible que el paciente posea una buena respuesta documentada a levodopa o apomorfina y que cuente con una buena red de apoyo familiar. Esto último es necesario para garantizar un adecuado cuidado y manejo del dispositivo, así como de su colocación.

\section{Evaluación de la respuesta a la apomorfina}

La realización de una prueba aguda de apomorfina previa al inicio de la infusión continua no se considera indispensable, siempre y cuando se tenga previamente documentada una respuesta clara a la terapia de reemplazo dopaminérgica. Una excepción se considera aquel paciente que recibe dosis altas de levodopa (hasta $400 \mathrm{mg}$ por toma) sin respuesta clínica y que se sospeche se deba a problemas de motilidad y absorción gástrica ${ }^{12}$.

\section{Preparación de la apomorfina en infusión subcutánea continua}

A la fecha, la apomorfina se encuentra disponible en tres presentaciones para su uso clínico: jeringas prellenadas con apomorfina $5 \mathrm{mg} / \mathrm{ml}$ (10 ml), ámpulas de apomorfina de $10 \mathrm{mg} / \mathrm{ml}(2 \circ 5 \mathrm{ml})$ y pluma prellenada de apomorfina $10 \mathrm{mg} / \mathrm{ml}(3 \mathrm{ml})$.

Para preparar la solución de apomorfina es necesario cargar en una jeringa de $20 \mathrm{ml}$ el ámpula del medicamento y diluir con solución salina al $0.9 \%$; de esta manera se obtiene $20 \mathrm{ml}$ en total. Por ejemplo, en caso de utilizar un ámpula de $5 \mathrm{ml}$ (50 mg de apomorfina) se requiere aforar la jeringa con $15 \mathrm{ml}$ de solución salina para obtener un total de $20 \mathrm{ml}$; si se decidiera utilizar dos ámpulas de apomorfina, cada una de $5 \mathrm{ml}$, se requeriría diluir con $10 \mathrm{ml}$ de solución salina. Dado que las presentaciones pueden cambiar, siempre se debe revisar la información para prescribir actualizada.

\section{Colocación de la bomba de apomorfina en infusión subcutánea continua}

La bomba de infusión de apomorfina consta de un mecanismo donde se carga la jeringa con el medicamento y que permite regular la velocidad de la infusión. Adicionalmente se cuenta con un catéter que se conecta a la aguja, la cual se recomienda se coloque de manera subcutánea a 2-3 cm lateral de la cicatriz umbilical. Como en cualquier aplicación subcutánea, se requiere descartar que la piel tenga alguna lesión (zona de irritación o datos de infección local) y hacer limpieza en el área en la que se colocará la aguja con una torunda con alcohol. La aguja se introduce en un solo movimiento y se fija con un adhesivo; la bomba se coloca dentro de una bolsa con asa, para facilitar su traslado. Dado que las bombas de infusión se actualizan frecuentemente es indispensable consultar el manual de operación de la bomba a manejar. La infusión de apomorfina generalmente se utiliza $16 \mathrm{~h}$ al día (tiempo en el que el paciente está despierto). De tal forma que la bomba y la aguja se retiran por la noche al terminar la infusión. Cuando el paciente tiene periodos intensos de OFF durante la noche, la infusión se utiliza 
Rev Mex Neuroci. 2019;20

Tabla 1. Diluciones sugeridas para el uso de apomorfina en infusión subcutánea con ampolleta de $50 \mathrm{mg} / 5 \mathrm{ml}$ (10 mg/ml) Requerimiento diario menor a $\mathbf{5 0} \mathbf{~ m g}$ de apomorfina

$5 \mathrm{ml}$ de apomorfina (una ampolleta de $10 \mathrm{mg} / \mathrm{ml}$ ) diluidos con $15 \mathrm{ml}$ de solución salina (0.9\%) para lograr una concentración de

$2.5 \mathrm{mg} / \mathrm{ml}(50 \mathrm{mg} / 20 \mathrm{ml})$.

Ejemplo: si se requiere una dosis de $1 \mathrm{mg} / \mathrm{h}$, entonces la velocidad de infusión será de $0.40 \mathrm{ml} / \mathrm{h}$

Requerimiento diario mayor a $\mathbf{5 0} \mathrm{mg}$ de apomorfina

$10 \mathrm{ml}$ de apomorfina (dos ampolletas de $10 \mathrm{mg} / \mathrm{ml}$ ) diluidos con $10 \mathrm{ml}$ de solución salina (0.9\%) para lograr una concentración de $5 \mathrm{mg} / \mathrm{ml}(100 \mathrm{mg} / 20 \mathrm{ml})$.

Ejemplo: si se requiere una dosis de $1 \mathrm{mg} / \mathrm{h}$, entonces la velocidad de infusión será de $0.20 \mathrm{ml} / \mathrm{h}$

$24 \mathrm{~h}^{13}$. Una vez colocada la bomba de infusión es indispensable no mojar el dispositivo, el resto de las actividades de la vida diaria las podrá realizar el paciente sin ninguna contraindicación. Se recomienda que el paciente se bañe antes de colocar la bomba para evitar que esta se pueda dañar con el agua.

\section{INICIO Y DOSIFICACIÓN DE LA APOMORFINA EN INFUSIÓN SUBCUTÁNEA CONTINUA}

Antes de iniciar la infusión continua de apomorfina se requiere premedicar al paciente con antieméticos con la finalidad de disminuir el riesgo de náuseas y vómito. El medicamento más sugerido en la literatura es la domperidona vía oral a dosis de $10 \mathrm{mg}$ administrada dos a tres veces al día, iniciando al menos $48 \mathrm{~h}$ antes del inicio de la apomorfina ${ }^{14} \mathrm{y}$ administrando una dosis una hora antes del inicio de la infusión de esta. Se debe recordar que la domperidona puede tener efectos adversos cardíacos severos tales como taquiarritmias, por lo que se deberá ser cauteloso con su uso en dosis mayores de $30 \mathrm{mg} / \mathrm{día}$; se sugiere fuertemente realizar un electrocardiograma en reposo antes del inicio de domperidona para descartar QT largo, taqui o bradiarritmias, y extrasistolia auricular 0 ventricular ${ }^{15}$. Así mismo, se sugiere realizar una biometría hemática y prueba de Coombs para excluir anemia hemolítica preexistente, dado que existe un riesgo bajo, pero presente, de anemia hemolítica con la administración de apomorfina. Finalmente, dado el efecto hipotensor de la apomorfina, debe llevarse a cabo un monitoreo de la presión arterial antes y durante la infusión del medicamento al inicio de la terapia.

Se recomienda que la infusión se inicie a $1 \mathrm{mg} / \mathrm{h}$ con incrementos de $0.5 \mathrm{mg} / \mathrm{h}$ cada 3-4 h. Así mismo, se recomienda no exceder la dosis total de $100 \mathrm{mg} / 24 \mathrm{~h}$. La infusión se debe titular con base en la respuesta clínica del paciente y la tolerancia al medicamento. Para calcular los requerimientos de apomorfina del paciente se debe realizar un cálculo total de dosis equivalentes de levodopa (con todos los fármacos dopaminérgicos que utilizaba el paciente previamente al inicio de la infusión de apomorfina) ${ }^{16}$. Esta dosis equivalente calculada se divide entre 10 para obtener la equivalencia aproximada con apomorfina ${ }^{11}$. Como ya se mencionó, se recomienda la infusión para las horas al día en las cuales el paciente esta despierto, que usualmente son $16 \mathrm{~h}$. En la tabla 1 se presentan recomendaciones para la dilución del medicamento en la terapia de AISC.

Un punto final que considerar es que con la terapia de infusión de apomorfina subcutánea es recomendable suspender de manera paulatina la terapia con agonistas dopaminérgicos antes del inicio de la infusión, aproximadamente en el transcurso de una semana ${ }^{17}$. Los inhibidores de la monoamino oxidasa-B (MAO-B), los inhibidores de la catecol O-metiltransferasa (COMT) y la amantadina también deben ser suspendidos de manera gradual antes del inicio de la terapia con apomorfina ${ }^{17}$, de tal manera que al momento de iniciar la AISC solo se esté recibiendo levodopa. Las dosis de levodopa se irán reduciendo conforme se incrementa la dosis de apomorfina, sobre todo si el paciente presenta discinesias.

\section{Contraindicaciones de la apomorfina en INFUSIÓN SUBCUTÁNEA CONTINUA}

Las contraindicaciones para la AISC incluyen aquellas relacionadas con el perfil de efectos adversos del medicamento. Estas contraindicaciones incluyen insuficiencia renal, cardiopatía descompensada, hipotensión ortostática severa, alucinaciones y psicosis severa, depresión respiratoria e hipersensibilidad conocida al metabisulfito sódico. No obstante, otras contraindicaciones incluyen una mala red de apoyo social y familiar 0 alguna otra condición que dificulte o impida el manejo adecuado de la bomba de infusión. 


\section{Perfil de seguridad de la apomorfina en INFUSIÓN SUBCUTÁNEA CONTINUA}

El efecto adverso más frecuente asociado a la infusión subcutánea de apomorfina es la aparición de nódulos cutáneos, los cuales se presentan prácticamente en la totalidad de los pacientes ${ }^{18}$. Otros efectos adversos incluyen sedación, somnolencia e hipersomnolencia diurna. También pueden presentarse náuseas, vómitos e hipotensión ortostática, aunque habitualmente pueden ser controlados. El desarrollo de alucinaciones es controvertido, con algunos autores que sugieren un riesgo bajo ${ }^{19}$. Un caso similar se presenta con el trastorno de control de impulsos, un estudio reciente concluye que la apomorfina es de los agonistas dopaminérgicos menos asociados con este síntoma ${ }^{20}$. Finalmente, la anemia hemolítica es un efecto adverso raro y reversible, pero que debe considerarse tanto al inicio como durante la terapia ${ }^{21}$.

Manejo de efectos adversos de la apomorfina en infusión subcutánea continua

Con la finalidad de reducir los nódulos cutáneos se recomienda hacer una buena higiene de la piel, rotar sitios de aplicación, usar agujas de teflón y cambiarlas en cada aplicación; también se recomienda dar un masaje en el sitio de la infusión posterior al retiro de la aguja22. La hipotensión ortostática puede ser tratada mediante el incremento de la ingesta de líquido y sal, el uso de medias compresivas y cambios lentos de postura; en caso necesario se puede utilizar mineralocorticoides como la fludrocortisona. Recientemente se ha reportado un efecto beneficioso de la domperidona en el tratamiento de hipotensión arterial asociada a agonistas dopaminérgicos ${ }^{23}$. Como ya se mencionó, para el tratamiento de las náuseas se recomienda la administración de domperidona, sin olvidar que el uso prolongado de este medicamento se ha asociado a prolongación del QT. La anemia hemolítica habitualmente es reversible con la suspensión de la infusión, pero es recomendable la valoración por un hematólogo ${ }^{22}$.

\section{EFECTIVIDAD DE LA APOMORFINA EN INFUSIÓN SUBCUTÁNEA CONTINUA SOBRE LOS SÍNTOMAS MOTORES}

La efectividad de la AISC ha sido demostrada desde los años ochenta con disminución del tiempo en OFF ${ }^{24,25}$. Más recientemente, el estudio TOLEDO, diseñado para evaluar la eficacia de la infusión de apomorfina subcutánea en los periodos de OFF en pacientes con EP en comparación con placebo, reportó una reducción de los periodos de OFF a las 12 semanas de $2.47 \mathrm{~h}$, en comparación a las $0.58 \mathrm{~h}$ del grupo placebo ${ }^{26}$. Otro estudio multicéntrico cuyo objetivo fue evaluar la eficacia de la AISC a largo plazo (5 años) reportó una reducción del tiempo de OFF del $79.51 \%$, una reducción de la intensidad de las discinesias del $32.1 \%$, y una reducción de la dosis diaria de levodopa $32.9 \%$. Un estudio en 230 pacientes a lo largo de 10 años reportó una disminución de $4 \mathrm{~h}$ en el tiempo en OFF en el grupo que permaneció activo; así mismo, no hubo incremento en las discinesias ni alteración en la cognición ${ }^{28}$. En la tabla 2 se resumen la población de estudio y resultados principales de los estudios antes mencionados.

\section{EFECTIVIDAD DE LA APOMORFINA EN INFUSIÓN SUBCUTÁNEA CONTINUA SOBRE LOS SÍNTOMAS NO MOTORES}

Existen hasta la fecha pocos estudios encaminados a evaluar el efecto de la AISC en la sintomatología no motora, pero los estudios enfocados en beneficio de fluctuaciones motores han reportado beneficio agregado para síntomas no motores que permite sugerir que la terapia con apomorfina subcutánea puede mejorar síntomas no motores tales como: insomnio, apatía, anhedonia, ansiedad, síndrome de piernas inquietas, urgencia urinaria, salivación y estreñimiento ${ }^{29,30}$. Se ha comparado la terapia con apomorfina frente a levodopa en el tratamiento de síntomas neuropsiquiátricos en EP reportando que los pacientes en tratamiento con apomorfina tienen una reducción del $45 \%$ en la escala de depresión de Beck en comparación con el grupo de levodopa, el cual incluso aumentó su puntuación ${ }^{31}$.

\section{Levodopa intestinal en gel}

Como es conocido, la estimulación pulsátil que se crea con la administración de levodopa oral en tabletas aunada a un vaciamiento gástrico errático ocasiona niveles plasmáticos fluctuantes de dopamina, que a su vez conducen a cambios en receptores dopaminérgicos, gabaérgicos y glutamatérgicos, lo que origina fluctuaciones motoras y discinesias ${ }^{32}$. Como consecuencia, surgió la necesidad de obtener una manera viable de administrar levodopa directamente en el duodeno. En 1986, Kurlan, et al. realizaron los primeros estudios con levodopa administrada en infusión. Lo lograron mediante una sonda nasoduodenal por la cual se administraba una 
Tabla 2. Características de la población de estudio y resultados del desenlace principal en los estudios de apomorfina en infusión subcutánea

\begin{tabular}{|c|c|c|}
\hline Estudio & Características del estudio & Resultados \\
\hline Stibe, et al., $1988^{24}$ & $\begin{array}{l}11 \text { sujetos con una edad media de } 56 \text { años (rango: } 32-70 \text { ) y } \\
\text { duración media de la enfermedad de } 14.4 \text { años (rango: } \\
\text { 9-20), duración media de tratamiento con levodopa de } 13.5 \\
\text { años (rango: } 9-17 \text { ) y dosis media inicial de levodopa de } \\
\text { 1,073 mg (rango: } 300-2,400 \text { ) }\end{array}$ & $\begin{array}{l}\text { La media de horas en OFF por día se } \\
\text { redujo de } 10.1 \text { a } 3.8 \text { (media de reducción: } \\
6.3 \pm 2 \mathrm{~h} \text { ) }\end{array}$ \\
\hline Chaudhuri, et al., $1988^{25}$ & $\begin{array}{l}9 \text { sujetos con edad media de } 59 \text { años y duración de la } \\
\text { enfermedad de } 17 \text { años (rango: 12-20), dosis diarias de } \\
\text { levodopa de } 886 \text { mg (rango: } 350-1,625 \text { ) y horas diarias en } \\
\text { OFF de } 7.3 \text { (rango: } 4-10 \text { ) }\end{array}$ & $\begin{array}{l}\text { La mejoría fue del } 85 \% \text {, reduciendo el } \\
\text { tiempo en OFF a } 1.1 \mathrm{~h}\end{array}$ \\
\hline García-Ruiz, et al., $2008^{27}$ & $\begin{array}{l}82 \text { sujetos con una edad media de } 67 \text { años (rango: } 23-85 \text { ) o } \\
\text { duración de la enfermedad de } 14.3 \pm 5.7 \text { años }\end{array}$ & $\begin{array}{l}\text { Se observa una reducción de las horas } \\
\text { en OFF de } 6.64 \pm 3.09 \text { a } 1.36 \pm 1.42 \text { h por } \\
\text { día }\end{array}$ \\
\hline Sesar, et al., $2017^{28}$ & $\begin{array}{l}230 \text { sujetos con una edad media de } 66.8 \pm 9.3 \text { años y } \\
\text { duración de la enfermedad de } 13 \pm 6.6 \text { años }\end{array}$ & $\begin{array}{l}\text { Reducción significativa de las horas } \\
\text { diarias en OFF de } 5.4 \pm 2.8 \text { a } 1.2 \pm 1.3\end{array}$ \\
\hline TOLEDO, $2018^{26}$ & $\begin{array}{l}107 \text { sujetos asignados aleatoriamente a } \\
\text { apomorfina }(n=53) \text { o a placebo }(n=54) \text {. Duración de la } \\
\text { enfermedad de } 11.2 \text { años y media de dosis diaria de } \\
\text { levodopa de } 954 \mathrm{mg} / \mathrm{dl}\end{array}$ & $\begin{array}{l}\text { Media de cambio en el tiempo en OFF } \\
\text { de }-2.47 \pm 3.7 \mathrm{~h} \text { por día en el grupo de } \\
\text { apomorfina y de }-0.58 \pm 2.8 \mathrm{~h} \text { por día para } \\
\text { el grupo placebo }\end{array}$ \\
\hline
\end{tabular}

infusión de levodopa preparada disolviendo las tabletas de levodopa/carbidopa más ácido ascórbico al $2 \%$ en agua estérili3. Posteriormente, el mismo grupo demostró que la entrega continua duodenal de levodopa era más eficiente, en comparación con la gástrica, a la duodenal intermitente y a las presentaciones orales de liberación prolongada en términos de farmacocinética ${ }^{34}$.

\section{Farmacología de levodopa intestinal en gel}

La presentación del medicamento, actualmente, es mediante un cassette o cartucho que contiene levodopa $(20 \mathrm{mg} / \mathrm{ml})$ más carbidopa $(5 \mathrm{mg} / \mathrm{ml})$ en una mezcla de carboximetil celulosa. Los cassettes contienen $100 \mathrm{ml}$, lo que en general cubre la dosis diaria total. El medicamento debe mantenerse a una temperatura de $2-8^{\circ} \mathrm{C}$. Para administrarlo es necesario colocar antes una sonda de yeyunostomía transgástrica endoscópica percutánea, esta se conecta a la bomba de infusión.

La LCIG posee la misma biodisponibilidad que la levodopa en comprimidos (81-98\%) con la distinción de que la velocidad de vaciado gástrico no influye en la tasa de absorción en intestino delgado, por lo que la variación intrasujeto de las concentraciones de levodopa no son significativas $^{35}$. Con la LCIG, los niveles plasmáticos se alcanzan en 10-30 min aproximadamente. La vida media de la levodopa intestinal en gel es de 1 a $2 \mathrm{~h}$. Así mismo, es importante mencionar que con LCIG no se ha observado desarrollo de tolerancia, es decir, la dosis se mantiene estable durante largos periodos de tiempo ${ }^{36-38}$.

\section{Vía de administración}

La vía de administración de la LCIG es necesariamente duodenal, ya sea por medio de una sonda nasoduodenal o bien mediante una yeyunostomía transgástrica.

\section{Indicaciones y selección del paciente}

Las indicaciones terapéuticas para la levodopa intestinal en gel incluyen las siguientes condiciones ${ }^{39}$ :

- Pacientes con EP avanzada con fluctuaciones motoras incapacitantes y que no se logran controlar adecuadamente con la optimización del tratamiento oral. - Ausencia de demencia o deterioro cognitivo severo. - Buena respuesta documentada a levodopa.

- Red de apoyo familiar adecuada.

\section{Evaluación de la respuesta a la levodopa}

Al igual que en cualquier otra de las terapias avanzadas asistidas por dispositivo, el requisito indispensable es una buena respuesta a la levodopa. Dado que la terapia está indicada en pacientes con enfermedad avanzada, difícilmente se tendrá que comprobar la respuesta a la terapia dopaminérgica. En caso necesario, 
Tabla 3. Esquema sugerido de dosificación de la terapia con levodopa/carbidopa intestinal en gel

\begin{tabular}{|c|c|}
\hline \multicolumn{2}{|c|}{ Levodopa/carbidopa intestinal en gel $(20 / 5 \mathrm{mg} / \mathrm{ml})$} \\
\hline Dosis matutina & $\begin{array}{l}\text { La dosis se basa en la dosis previa de levodopa oral que tomaba el paciente } \\
\text { La dosis recomendada es el equivalente al } 80 \% \text { de la toma matutina previa de levodopa oral }\end{array}$ \\
\hline Dosis continua de mantenimiento & $\begin{array}{l}\text { La dosis se calcula de acuerdo a la ingesta diaria previa de levodopa oral } \\
\text { A dicha dosis se debe restar la dosis matutina y administrar el } 90 \% \text { en infusión continua durante } 16 \mathrm{~h} \\
\text { La dosis de mantenimiento se puede titular en intervalos de } 2 \mathrm{mg} / \mathrm{h}(0.1 \mathrm{ml} / \mathrm{h}) \\
\text { Si se suspenden otros medicamentos dopaminérgicos, la dosis de la levodopa/carbidopa } \\
\text { intestinal en gel debe ajustarse calculando la dosis diaria de equivalentes de levodopa (LEDD) }\end{array}$ \\
\hline Dosis extra en bolo & $\begin{array}{l}\text { Se utiliza en caso de periodos de hipocinesia severa } \\
\text { La dosis extra en bolo se debe ajustar de forma individual, habitualmente es de } 0.5 \text { a } 2.0 \mathrm{ml}\end{array}$ \\
\hline
\end{tabular}

la respuesta puede evaluarse mediante un reto agudo de levodopa habitual. Nuevamente es importante mencionar que en el caso de una pobre respuesta debe descartarse la posibilidad de alteraciones en la absorción del medicamento como consecuencia de alteraciones gastrointestinales, en cuyo caso una prueba aguda de apomorfina puede ser de mayor utilidad ${ }^{40}$.

Por otra parte, la mayor parte de centros especializados realizan una prueba terapéutica de la LCIG a través de una sonda nasoyeyunal previamente a la gastrostomía ${ }^{41}$. Esta prueba permite evaluar la respuesta y la tolerabilidad, y permite al paciente familiarizarse con la bomba y el sistema de infusión. No obstante, existen reportes que han sugerido que esta prueba puede omitirse $^{42}$. En caso de realizar la prueba, el paciente debe hospitalizarse y la colocación de la sonda se hace de forma habitual, ya sea por migración pasiva, por fluoroscopia o por endoscopia bajo sedación. El cálculo de la dosis de LCIG se explica más adelante, pero se debe considerar que $1 \mathrm{ml}$ de levodopa intestinal en gel equivale a $20 \mathrm{mg}$ de levodopa ${ }^{43}$. El tratamiento debe iniciarse por la mañana en OFF, y la infusión mantenerse de 12 a $16 \mathrm{~h}$. Tras evaluar la respuesta objetiva y subjetiva, la tolerabilidad, el entendimiento del funcionamiento por parte del paciente y familiares, y sobre todo las expectativas del, se puede proceder a la gastrostomía.

\section{Colocación de la bomba de la levodopal carbidopa intestinal en gel}

El método de colocación es la gastrostomía endoscópica percutánea con sonda yeyunal (PEG-J, por sus siglas en inglés) $)^{44}$. El procedimiento de PEG-J se lleva a cabo de forma habitual. Si se realizó previamente la prueba con sonda nasoyeyunal se continua con la dosificación, en caso contrario debe seguirse el procedimiento que se describe a continuación.

\section{Inicio y dosificación de la levodopa/ carbidopa intestinal en gel}

Para iniciar la terapia con la LCIG, ya sea por sonda en la fase de prueba o por PEG-J, es necesario hacer el cálculo total de levodopa oral. Con base en lo anterior se calculan tres dosis distintas: la dosis matutina, la dosis de infusión continua y la dosis extra ${ }^{43}$.

En la dosis matutina se administra el equivalente al $80 \%$ de la dosis matutina de levodopa oral previa. Por ejemplo, si el paciente recibía 200 mg de levodopa por la mañana, entonces se administrarán $160 \mathrm{mg}$ de levodopa intestinal en gel. La reducción de dosis se realiza para disminuir el riesgo de discinesias. Para la dosis continua, se resta la dosis matutina de la dosis diaria total de levodopa oral y se administra el 90\% en 16 h de infusión. Por ejemplo, si el paciente recibía $1,500 \mathrm{mg}$ de levodopa al día, se le restan los $160 \mathrm{mg}$ de la dosis de la mañana, restando $1,340 \mathrm{mg}$ a pasar en $16 \mathrm{~h}$. Como se mencionó previamente, la conversión es de $1 \mathrm{ml}$ a $20 \mathrm{mg}$, por lo que se requieren $67 \mathrm{ml} / 16 \mathrm{~h}$ o $4.2 \mathrm{ml} / \mathrm{h}$. Finalmente, las dosis extras se pueden administrar en bolos de $0.5-2 \mathrm{ml}$ según los requerimientos del paciente por periodos de hipocinesia. En caso de que se requieran más de cinco bolos extras al día, entonces se sugiere un ajuste de dosis ${ }^{45}$.

La titulación sugerida depende de la velocidad de infusión. En la tabla 3 se resume el esquema sugerido de dosificación de la terapia y en la tabla 4 se muestran los esquemas sugeridos para la dosificación y titulación de la LCIG. En general, se sugiere que todo el procedimiento se realice de forma intrahospitalaria, sin embargo, también se han propuesto modelos ambulatorios ${ }^{46}$.

\section{Contraindicaciones de la levodopal carbidopa intestinal en gel}

Las contraindicaciones para esta opción terapéutica incluyen principalmente demencia severa y psicosis 
Tabla 4. Esquema sugerido para la dosificación y titulación de la levodopa/carbidopa intestinal en gel de acuerdo con la respuesta y efectos secundarios

\begin{tabular}{|l|c|c|c|}
\hline Dosis por hora $(\mathrm{ml} / \mathrm{h})$ & Dosis matutina & Dosis de infusión continua (durante 16 h) & \\
\hline$<6 \mathrm{ml} / \mathrm{h}$ & $\uparrow 1.0 \mathrm{ml} / \mathrm{h}$ & $\uparrow 0.3 \mathrm{ml} / \mathrm{h}$ & $\downarrow 0.2 \mathrm{ml} / \mathrm{h}$ \\
\hline$>6 \mathrm{ml} / \mathrm{h}$ & $\uparrow 2.0 \mathrm{ml} / \mathrm{h}$ & $\uparrow 0.4 \mathrm{ml} / \mathrm{h}$ & $\downarrow 0.2 \mathrm{ml} / \mathrm{h}$
\end{tabular}

$\uparrow$ Incremento basado en la impresión clínica de una respuesta inadecuada.

$\downarrow$ Decremento basado en la presencia de discinesias y otros efectos adversos.

Tabla 5. Características de la población de estudio y resultados del desenlace principal en los estudios de apomorfina en infusión subcutánea

\begin{tabular}{|l|l|l|}
\hline Estudio & Características del estudio & Resultados \\
\hline Nyholm, et al., 200550 & $\begin{array}{l}\text { 24 sujetos en un diseño cruzado. La mediana de } \\
\text { edad fue de } 66 \text { años, mediana de duración de la } \\
\text { enfermedad de } 13 \text { años y } 13 \text { años de tratamiento } \\
\text { con levodopa oral }\end{array}$ & $\begin{array}{l}\text { La mediana del porcentaje en las escalas de ON } \\
\text { funcional aumento del } 81 \text { al } 100 \% \text { con la infusión. } \\
\text { Acompañado de una disminución en el tiempo en } \\
\text { OFF y sin cambio en el ON con discinesias molestas }\end{array}$ \\
\hline Fernández, et al., 201551 & $\begin{array}{l}272 \text { sujetos con una edad media de } 64.1 \pm 9.1 \text { años } \\
\text { y duración de la enfermedad de } 12.5 \pm 5.5 \text { años. } \\
\text { Dosis diaria de levodopa de } 1,082 \pm 582.1 \mathrm{mg} \text { y un } \\
\text { tiempo en OFF de } 6.75 \pm 2.35 \mathrm{~h} / \mathrm{d}\end{array}$ & $\begin{array}{l}\text { Disminución de la media del tiempo en OFF diario de } \\
\text { 4.4 } \mathrm{h}(65.6 \%) \text { y aumento del tiempo en ON sin } \\
\text { discinesias molestas de } 4.8 \mathrm{~h}(62.9 \%)\end{array}$ \\
\hline Wang, et al., 201852 & $\begin{array}{l}\text { Revisión sistemática y metaanálisis incluyendo 384 } \\
\text { sujetos }\end{array}$ & $\begin{array}{l}\text { Diferencias significativas en el tiempo en 0N sin } \\
\text { discinesias molestas (diferencia de medias } \\
\text { estandarizada de 0.55) y del tiempo en } \\
\text { OFF (diferencia de medias estandarizada de -1.19) }\end{array}$ \\
\hline
\end{tabular}

activa $^{47}$. En cuanto al procedimiento, se incluyen todas las contraindicaciones para la colocación de la PEG-J. Adicionalmente, al igual que con la AICS, una mala red de apoyo y pobre entendimiento del funcionamiento del dispositivo pueden contraindicar la terapia.

\section{Perfil de seguridad de la levodopal carbidopa intestinal en gel}

Los efectos adversos más comunes reportados con LCIG son: dolor abdominal, náuseas, pérdida de peso, estreñimiento, caídas, neuropatía, pérdida de peso y alucinaciones. Los efectos adversos reportados por el sistema de PEG-J son: dislocación, oclusión de la sonda, infección en el sitio de inserción de la sonda, rotura de la bomba de infusión, dolor abdominal y neumoperitone $0^{48}$.

\section{Manejo de efectos adversos de la levodopal carbidopa intestinal en gel}

El manejo de efectos adversos relacionados con el medicamento se resuelve con el ajuste de dosis. En el caso de la polineuropatía crónica asociada a esta terapia se ha recomendado la suplementación con vitamina B12 y ácido fólico ${ }^{49}$. En la tabla 4 se resumen otros posibles efectos adversos y complicaciones del procedimiento y tratamiento con LCIG.

\section{Efectividad de la levodopa/carbidopa intestinal en gel sobre los síntomas motores}

Al igual que en el caso de la apomorfina, existen múltiples estudios sobre la efectividad de esta terapia. Un estudio cruzado que comparó la LCIG como monoterapia frente a polifarmacia reportó una disminución de 53 a 35 puntos en en la escala unificada de la enfermedad de Parkinson (UPDRS) con el uso de la LCIG $^{50}$. Otro estudio con seguimiento a 12 meses demostró una disminución en el tiempo en OFF de $4.4 \mathrm{~h}$ $(65.6 \%)$ y un incremento en el tiempo en ON sin discinesias molestas de $4.8 \mathrm{~h}(62.9 \%)^{51}$. Un metaanálisis reciente utilizando la información de 384 pacientes reportó una disminución de $1.19 \mathrm{~h}$ en el tiempo en OFF, un incremento en el tiempo en ON sin discinesias molestas de $0.55 \mathrm{~h}$, sin cambios significativo en el tiempo en ON con discinesias molestas ${ }^{52}$. En la tabla 5 
Figura 1. Recomendaciones sugeridas para la selección de la mejor terapia asistida por dispositivo, de acuerdo con características generales y perfil de síntomas motores y no motores.

\begin{tabular}{|c|c|c|c|c|}
\hline & & $\begin{array}{l}\text { Apomorfina en infusión } \\
\text { continua subcutánea }\end{array}$ & $\begin{array}{l}\text { Levodopa/carbidopa } \\
\text { intestinal en gel }\end{array}$ & $\begin{array}{l}\text { Estimulación cerebral } \\
\text { profunda }\end{array}$ \\
\hline \multirow[t]{8}{*}{$\begin{array}{l}\text { Características } \\
\text { generales }\end{array}$} & Edad $>70$ años & & & \\
\hline & Múltiples comorbilidades & & & \\
\hline & Uso de anticoagulación & & & \\
\hline & Independencia & & & \\
\hline & $\begin{array}{l}\text { Pobre apoyo familiar y } \\
\text { social }\end{array}$ & & & \\
\hline & $\begin{array}{l}\text { Limitaciones de } \\
\text { capacidades técnicas }\end{array}$ & & & \\
\hline & Alto riesgo quirúrgico & & & \\
\hline & $\begin{array}{l}\text { Excelente respuesta a } \\
\text { levodopa }\end{array}$ & & & \\
\hline \multirow{6}{*}{$\begin{array}{l}\text { Síntomas } \\
\text { motores }\end{array}$} & Temblor & & & \\
\hline & Discinesias molestas & & & \\
\hline & Disartria & & & \\
\hline & Caídas frecuentes & & & \\
\hline & $\begin{array}{l}\text { Limitación de actividades de } \\
\text { la vida diaria }\end{array}$ & & & \\
\hline & Congelamiento en OFF & & & \\
\hline \multirow{7}{*}{$\begin{array}{l}\text { Síntomas no } \\
\text { motores }\end{array}$} & Demencia & & & \\
\hline & Deterioro cognitivo leve & & & \\
\hline & Psicosis & & & \\
\hline & Depresión & 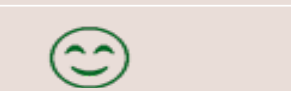 & & \\
\hline & $\begin{array}{l}\text { Trastorno del control de } \\
\text { impulsos }\end{array}$ & & & \\
\hline & Dolor & 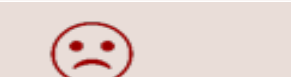 & & \\
\hline & Problemas de sueño & $\Leftrightarrow$ & 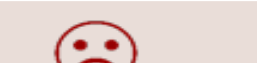 & 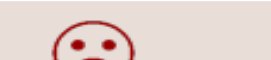 \\
\hline
\end{tabular}


Rev Mex Neuroci. 2019;20

se resumen las características principales y el desenlace primario de los estudios antes citados.

\section{Efectividad de la levodopa/carbidopa intestinal en gel sobre los síntomas no motores}

El estudio GLORIA, que incluyó 375 pacientes en 75 centros de trastornos del movimiento, demostró que además de los beneficios motores la terapia de LCIG mejora también síntomas no motores tales como: sueño, fatiga, estado de ánimo, problemas gastrointestinales y urgencia urinaria ${ }^{53}$. Sin embargo, se requieren mayores estudios en este respecto.

\section{Comparación de las terapias de infusión}

Como se ha descrito, ambas terapias de infusión se encuentran indicadas en pacientes con EP avanzada, al igual que la ECP. Consecuentemente, la pregunta más relevante es cuál es la terapia más efectiva; la respuesta es que todas las terapias asistidas por dispositivo son efectivas, en el paciente adecuado. Recientemente, Antonini, et al. realizaron un consenso sobre estas terapias ${ }^{54}$; las recomendaciones se resumen en la figura 1.

En forma simplista, las terapias de infusión están indicadas en pacientes con EP con periodos de OFF problemáticos o múltiples tomas de levodopa, aún en la presencia de algún grado de deterioro cognitivo importante. Este último criterio es relevante, ya que contraindica la opción de ECP 55 . Dadas las complicaciones metodológicas y económicas se carece de un estudio comparativo doble ciego sobre estas terapias; no obstante, el Eurolnf comparó en un diseño abierto la AISC y la LCIG, concluyendo que ambas terapias son efectivas en el control de síntomas motores, complicaciones motoras, calidad de vida y en algunos síntomas no motores ${ }^{56}$.

Otro punto que comentar es el costo-utilidad y el costo-efectividad de las terapias asistidas por dispositivo. Los costos por año de vida ajustado a calidad ganado son menores para el caso de la ECP, seguidos de la AISC, y significativamente mayores para la $\mathrm{LCIG}^{57}$. Por otra parte, la relación de costo-efectividad incremental (diferencia de costos entre un medicamento y su alternativa multiplicado por la diferencia entre los años de vida ajustados a calidad) de AISC es superior a la de la $L C I G$ e incluso que a la de la ECP ${ }^{58}$. En todo caso, la LCIG también es costo-efectiva con relación a la mejor terapia médica estándar en pacientes con enfermedad avanzada ${ }^{59}$. Se debe resaltar que los estudios de farmacoeconomía disponibles a la fecha se han realizado en países desarrollados, con sistemas de salud robustos y en los que tanto la AISC como la LCIG son catalogadas como fármacos huérfanos.

\section{Conclusión}

La AISC y la LCIG son terapias asistidas por dispositivo indicadas en personas con EP avanzada con respuesta insuficiente de la sintomatología motora y complicaciones motoras tras la optimización del tratamiento oral o transdérmico. La selección adecuada del paciente radica en el conocimiento y entendimiento de las indicaciones y contraindicaciones, beneficios motores y no motores, costos, y expectativa del paciente y su cuidador. Como con toda terapia existe riesgo de efectos adversos y complicaciones, pero su uso adecuado conlleva una mejora en la calidad de vida. En México la AISC ya cuenta con registro sanitario (076M2017) y también está disponible en otros países de América Latina, mientras que la LCIG se espera esté disponible en la región en un próximo futuro.

\section{Bibliografía}

1. Blin P, Dureau-Pournin C, Foubert-Samier A, Grolleau A, Corbillon E, Jové J, et al. Parkinson's disease incidence and prevalence assessment in France using the national healthcare insurance database. Eur J Neurol. 2015;22(3):464-71

2. Enders D, Balzer-Geldsetzer M, Riedel O, Dodel R, Wittchen HU, Sensken SC, et al. Prevalence, duration and severity of Parkinson's disease in Germany: A combined meta-analysis from literature data and outpatient samples. Eur Neurol. 2017;78(3-4):128-36.

3. Morgan JC, Fox SH. Treating the motor symptoms of Parkinson disease Continuum (Minneap Minn). 2016;22(4 Movement Disorders):1064-85.

4. Luquin MR, Kulisevsky J, Martinez-Martin P, Mir P, Tolosa ES. Consensus on the definition of advanced Parkinson's disease: A neurologists-based Delphi Study (CEPA Study). Parkinsons Dis. 2017;2017:4047392.

5. Tomiyama M. Symptoms and pathophysiology of dyskinesias. Brain Nerve. 2017;69:1409-16.

6. Timpka J, Nitu B, Datieva V, Odin P, Antonini A. Device-aided treatment strategies in advanced Parkinson's disease. Int Rev Neurobiol. 2017;132:453-74.

7. Djamshidian A, Poewe W. Apomorphine and levodopa in Parkinson's disease: Two revolutionary drugs from the 1950's. Parkinsonism Relat Disord. 2016;33:S9-12

8. Taba P, Lees A, Stern G. Erich Harnack (1852-1915) and a short history of apomorphine. Eur Neurol. 2013;69(6):321-4.

9. Przedborski S1, Levivier M, Raftopoulos C, Naini AB, Hildebrand J. Peripheral and central pharmacokinetics of apomorphine and its effect on dopamine metabolism in humans. Mov Disord. 1995;10(1):28-36.

10. Ostergaard L, Werdelin L, Odin P, Lindvall O, Dupont E, Christensen PB, et al. Pen injected apomorphine against off phenomena in late Parkinson's disease: a double blind, placebo controlled study. J Neurol Neurosurg Psychiatry. 1995;58(6):681-7.

11. Trenkwalder $C$, Chaudhuri KR, García Ruiz PJ, LeWittP, Katzenschlager $R$ Sixel-Döring $F$, et al.; Expert Consensus Group for Use of Apomorphine in Parkinson's Disease, Baker M, Ceballos-Baumann A, Deuschl G, Drapier S, Ebersbach G, Evans A, et al. Expert Consensus Group report on the use of apomorphine in the treatment of Parkinson's disease--Clinical practice recommendations. Parkinsonism Relat Disord. 2015; 21(9):1023-30.

12. Bhidayasiri $R$, Chaudhuri KR, LeWitt $P$, Martin A, Boonpang $K$, van Laar T. Effective delivery of apomorphine in the management of Parkinson disease: practical considerations for clinicians and Parkinson nurses. Clin Neuropharmacol. 2015;38(3):89-103. 
13. Fernández-Pajarín G, Sesar Á, Ares B, Castro A. Evaluating the efficacy of nocturnal continuous subcutaneous apomorphine infusion in sleep disorders in advanced Parkinson's disease: The APO-NIGHT Study. J Parkinsons Dis. 2016:6(4):787-92.

14. Arnold G, Schwarz J, Macher C, Oertel WH. Domperidone is superior to ondansetron in acute apomorphine challenge in previously untreated parkinsonian patients - A double blind study. Parkinsonism Relat Disord. 1997;3(4):191-3.

15. Lertxundi U, Domingo-Echaburu S, Soraluce A, García M, Ruiz-Osante B, Aguirre C. Domperidone in Parkinson's disease: a perilous arrhythmogenic or the gold standard? Curr Drug Saf. 2013;8(1):63-8.

16. Tomlinson CL, Stowe R, Patel S, Rick C, Gray R, Clarke CE. Systematic review of levodopa dose equivalency reporting in Parkinson's disease. Mov Disord. 2010;25(15):2649-53.

17. Moreno López CL, Bernal Pacheco O, Barrios Vincos G, Arango Uribe G, Cerquera Cleves C, Orozco Vélez JL, et al. Consenso de la Asociación Colombiana de Neurología sobre el uso de apomorfina en la enfermedad de Parkinson. Acta Neurol Colomb. 2018;34(1):25-39.

18. Grandas F. Subcutaneous infusions of apomorphine: a reappraisal of its therapeutic efficacy in advanced Parkinson's disease. Expert Rev Neurother. 2013;13(12):1343-53.

19. Borgemeester RW, Lees AJ, van Laar T. Parkinson's disease, visual hallucinations and apomorphine: A review of the available evidence. Parkinsonism Relat Disord. 2016;27:35-40.

20. Moore TJ, Glenmullen J, Mattison DR. Reports of pathological gambling hypersexuality, and compulsive shopping associated with dopamine receptor agonist drugs. JAMA Intern Med. 2014;174(12):1930-3.

21. Pietz $K$, Hagell $P$, Odin $P$. Subcutaneous apomorphine in late stage Parkinson's disease: a long term follow up. J Neurol Neurosurg Psychiatry. 1998;65(5):709-16

22. Bhidayasiri R, Garcia Ruiz PJ, Henriksen T. Practical management of adverse events related to apomorphine therapy. Parkinsonism Relat Disord. 2016;33(Suppl 1):S42-8.

23. Bacchi S, Chim I, Kramer P, Postuma RB. Domperidone for hypotension in Parkinson's disease: A systematic review. J Parkinsons Dis. 2017; 7(4):603-17.

24. Stibe CM, Lees AJ, Kempster PA, Stern GM. Subcutaneous apomorphine in parkinsonian on-off oscillations. Lancet. 1988;1(8582):403-6.

25. Chaudhuri KR, Critchley P, Abbott RJ, Pye IF, Millac PA. Subcutaneous apomorphine for on-off oscillations in Parkinson's disease. Lancet. 1988;2(8622):1260

26. Katzenschlager R, Poewe W, Rascol O, Trenkwalder C, Deuschl G Chaudhuri KR, et al. Apomorphine subcutaneous infusion in patients with Parkinson's disease with persistent motor fluctuations (TOLEDO): a multicentre, double-blind, randomised, placebo-controlled trial. Lancet Neurol. 2018;17(9):749-59.

27. García Ruiz PJ, Sesar Ignacio A, Ares Pensado B, Castro García A, Alonso Frech F, Alvarez López M, et al. Efficacy of long-term continuous subcutaneous apomorphine infusion in advanced Parkinson's disease with motor fluctuations: a multicenter study. Mov Disord. 2008;23(8):1130-6.

28. Sesar Á, Fernández-Pajarín G, Ares B, Rivas MT, Castro A. Continuous subcutaneous apomorphine infusion in advanced Parkinson's disease: 10-year experience with 230 patients. J Neurol- 2017;264(5):946-54.

29. Rosa-Grilo M, Qamar MA, Evans A, Chaudhuri KR. The efficacy of apomorphine - A non-motor perspective. Parkinsonism Relat Disord. 2016;33 Suppl 1:S28-35.

30. Todorova A, Ray Chaudhuri K. Subcutaneous apomorphine and non-motor symptoms in Parkinson's disease. Parkinsonism Relat Disord. 2013; 19(12):1073-8.

31. Morgante L, Basile G, Epifanio A, Spina E, Antonini A, Stocchi F, et al. Continuous apomorphine infusion (CAI) and neuropsychiatric disorders in patients with advanced Parkinson's disease: a follow-up of two years Arch Gerontol Geriatr Suppl. 2004;(9):291-6.

32. Bestetti A, Capozza A, Lacerenza M, Manfredi L, Mancini F. Delayed gastric emptying in advanced Parkinson disease: Correlation with therapeutic doses. Clin Nucl Med. 2017;:42(2):83-7.

33. Kurlan R, Rubin AJ, Miller C, Rivera-Calimlim L, Clarke A, Shoulson I Duodenal delivery of levodopa for on-off fluctuations in parkinsonism: preliminary observations. Ann Neurol. 1986;20(2):262-5.

34. Kurlan R, Nutt JG, Woodward WR, Rothfield K, Lichter D, Miller C et al. Duodenal and gastric delivery of levodopa in parkinsonism. Ann Neurol. 1988;23(6):589-95.

35. Othman AA, Dutta S. Population pharmacokinetics of levodopa in subjects with advanced Parkinson's disease: levodopa-carbidopa intestinal gel infusion vs. oral tablets. Br J Clin Pharmacol. 2014;78(1):94-105.

36. Palhagen SE, Sydow O, Johansson A, Nyholm D, Holmberg B, Widner H, et al. Levodopa-carbidopa intestinal gel (LCIG) treatment in routine care of patients with advanced Parkinson's disease: An open-label prospective observational study of effectiveness, tolerability and healthcare costs. Parkinsonism Relat Disord. 2016;29:17-23.

37. Nyholm D, Klangemo K, Johansson A. Levodopa/carbidopa intestinal gel infusion long-term therapy in advanced Parkinson's disease. Eur J Neurol. 2012; 19:1079-85
38. Zibetti M, Merola A, Ricchi V, Marchisio A, Artusi CA, Rizzi L, et al. Long-term duodenal levodopa infusion in Parkinson's disease: a 3-year motor and cognitive follow-up study. J Neurol. 2013;260:105-14.

39. Devos D; French DUODOPA Study Group. Patient profile, indications, efficacy and safety of duodenal levodopa infusion in advanced Parkinson's disease. Mov Disord. 2009; 24(7):993-1000.

40. Albanese A, Bonuccelli U, Brefel C, Chaudhuri KR, Colosimo C, Eichhorn $\mathrm{T}$, et al. Consensus statement on the role of acute dopaminergic challenge in Parkinson's disease. Mov Disord. 2001;16(2):197-201.

41. Lew MF, Slevin JT, Krüger R, Martínez Castrillo JC, Chatamra K, Dubow JS, et al. Initiation and dose optimization for levodopa-carbidopa intestinal gel: Insights from phase 3 clinical trials. Parkinsonism Relat Disord. 2015; 21(7):742-8.

42. Santos-Garcia D, de Deus-Fonticoba T. Initiation of levodopa entera infusion: is always the test with nasojejunal tube really necessary? Rev Neurol. 2018;67(5):192-3.

43. Santos García D, Martínez Castrillo JC, Puente Périz V, Seoane Urgorri A, Fernández Díez S, Benita León V, et al. Clinical management of patients with advanced Parkinson's disease treated with continuous intestinal infusion of levodopa/carbidopa. Neurodegener Dis Manag. 2016; 6(3):187-202.

44. Cheron J, Deviere J, Supiot F, Ballarin A, Eisendrath P, Toussaint E, et al. The use of enteral access for continuous delivery of levodopa-carbidopa in patients with advanced Parkinson's disease. United European Gastroenterol J. 2017;5(1):60-8.

45. Pedersen SW, Clausen J, Gregerslund MM. Practical guidance on how to handle levodopa/carbidopa intestinal gel therapy of advanced PD in a movement disorder clinic. Open Neurol J. 2012;6:37-50.

46. Fasano A, Liu LW, Poon YY, Lang AE. Initiating intrajejunal infusion of levodopa/carbidopa intestinal gel: an outpatient model. Mov Disord. 2015; 30(4):598-99.

47. Catalán MJ, Antonini $A$, Calopa $M$, Bajenaru $O$, de Fábregues $O$, Mínguez-Castellanos A, et al. Can suitable candidates for levodopa/ carbidopa intestinal gel therapy be identified using current evidence? eNeurologicalSci. 2017:8:44-53.

48. Olanow CW, Kieburtz K, Odin P, Espay AJ, Standaert DG, Fernandez HH, et al.; LCIG Horizon Study Group. Continuous intrajejunal infusion of levodopa-carbidopa intestinal gel for patients with advanced Parkinson's disease: a randomised, controlled, double-blind, double-dummy study. Lancet Neurol. 2014;13(2):141-9.

49. Sensi M, Cossu G, Mancini F, Pilleri M, Zibetti M, Modugno N, et al.; Italian levodopa carbidopa intestinal gel working group. Which patients discontinue? Issues on levodopa/carbidopa intestinal gel treatment: Italian multicentre survey of 905 patients with long-term follow-up. Parkinsonism Relat Disord. 2017;38:90-2

50. Nyholm D, Nilsson Remahl Al, Dizdar N, Constantinescu R, Holmberg B Jansson $\mathrm{R}$, et al. Duodenal levodopa infusion monotherapy vs oral polypharmacy in advanced Parkinson disease. Neurology. 2005;64(2):216-23.

51. Fernandez HH, Standaert DG, Hauser RA, Lang AE, Fung VS, Klostermann $F$, et al. Levodopa-carbidopa intestinal gel in advanced Parkinson's disease: final 12-month, open-label results. Mov Disord. 2015;30(4):500-9.

52. Wang L, Li J, Chen J. Levodopa-carbidopa intestinal gel in Parkinson's disease: A systematic review and meta-analysis. Front Neurol. 2018; 30:9:620.

53. Antonini A, Yegin A, Preda C, Bergmann L, Poewe W; GLORIA study investigators and coordinators. Global long-term study on motor and non-motor symptoms and safety of levodopa-carbidopa intestinal gel in routine care of advanced Parkinson's disease patients; 12-month interim outcomes. Parkinsonism Relat Disord. 2015;21(3):231-5.

54. Antonini A, Stoessl AJ, Kleinman LS, Skalicky AM, Marshall TS, Sail KR, et al. Developing consensus among movement disorder specialists on clinical indicators for identification and management of advanced Parkinson's disease: a multi-country Delphi-panel approach. Curr Med Res Opin. 2018;20:1-11

55. Odin P, Ray Chaudhuri K, Slevin JT, Volkmann J, Dietrichs E, Martinez-Martin $\mathrm{P}$, et al.; National Steering Committees. Collective physician perspectives on non-oral medication approaches for the management of clinically relevant unresolved issues in Parkinson's disease: Consensus from an international survey and discussion program. Parkinsonism Relat Disord. 2015;21(10):1133-44.

56. Martinez-Martin P, Reddy P, Katzenschlager R, Antonini A, Todorova A, Odin $\mathrm{P}$, et al. Eurolnf: a multicenter comparative observational study of apomorphine and levodopa infusion in Parkinson's disease. Mov Disord. 2015;30(4):510-6.

57. Vivancos-Matellano F, Garcia-Ruiz AJ, Garcia-Agua SN. Pharmacoeconomic study of the treatment of advanced Parkinson's disease. Rev Neurol. 2016:63(12):529-36.

58. Walter E, Odin P. Cost-effectiveness of continuous subcutaneous apomorphine in the treatment of Parkinson's disease in the UK and Germany. J Med Econ. 2015;18(2):155-65.

59. Lowin J, Sail K, Baj R, Jalundhwala $Y$, Marshall TS, Konwea H, et al. The cost-effectiveness of levodopa/carbidopa intestinal gel compared to standard care in advanced Parkinson's disease. J Med Econ. 2017;20(11):1207-15. 